\title{
Fluctuations in bovine ovarian follicular fluid composition throughout the oestrous cycle
}

\author{
Nicolas M Orsi ${ }^{1,2}$, Nadia Gopichandran ${ }^{1,2}$, Henry J Leese ${ }^{2}$, Helen M Picton ${ }^{3}$ \\ and Sarah E Harris ${ }^{2,3}$ \\ ${ }^{1}$ Perinatal Research Group, Department of Paediatrics, Obstetrics and Gynaecology, D Floor, Clarendon Wing, \\ Leeds General Infirmary, Belmont Grove, Leeds LS2 9NS, UK, ${ }^{2}$ Department of Biology, University of York, \\ PO Box 373, Heslington, York YO10 5YW, UK and ${ }^{3}$ Reproduction and Early Development Group, Department of \\ Paediatrics, Obstetrics and Gynaecology, D Floor, Clarendon Wing, Leeds General Infirmary, Belmont Grove, \\ Leeds LS2 9NS, UK \\ Correspondence should be addressed to N M Orsi; Email: n.m.orsi@leeds.ac.uk
}

\begin{abstract}
Bovine oocyte maturation in vitro frequently results in abnormal cytoplasmic maturation and failure to acquire developmental competence. This is, in part, likely to be due to the non-physiological nutritional milieu to which oocytes are exposed. Improvements in oocyte developmental potential may be achieved by modelling nutrient profiles on those of preovulatory follicular fluid (FF). However, little is known about fluctuations in FF nutrient levels according to follicle dominance and oestrous cyclicity. This study therefore characterised the carbohydrate and amino acid profile of FF according to these parameters, and compared preovulatory FF composition with that of maturation medium. Carbohydrate concentrations $(n=121)$ were determined enzymatically whilst amino acid profiles $(n=40)$ were determined by reverse-phase HPLC. Pyruvate and glucose concentrations were unaffected by follicle dominance, whereas Stage III-IV lactate profiles were higher in non-dominant FF $(P<\mathbf{0 . 0 1})$. While most dominant FF amino acid concentrations were affected by oestrous stage, only glutamate, alanine, leucine and lysine levels fluctuated in non-dominant FF. Glucose and lactate concentrations were significantly negatively correlated, whereas most amino acids were significantly positively correlated with each other. Maturation medium had higher pyruvate and lower lactate concentrations than preovulatory FF $(P<0.001)$, whereas glucose level was similar. All amino acid levels (except histidine, taurine, alanine and tryptophan) differed significantly between maturation medium and preovulatory FF. These data indicated that FF composition varies throughout the oestrous cycle. Preovulatory FF nutrient profile differed from that of maturation medium, perhaps accounting for the poor developmental competence of in vitro matured oocytes. These data may contribute to the formulation of a nutritionally more physiological maturation medium.
\end{abstract}

Reproduction (2005) 129 219-228

\section{Introduction}

Routine maturation of bovine oocytes in vitro underpins commercial embryo production and emerging biotechnologies such as cloning and transgenics (Kubota et al. 1998, Chen et al. 2002, Galli et al. 2003). However, current in vitro production protocols are inefficient at producing viable embryos (Lonergan et al. 2001), partly due to abnormal oocyte cytoplasmic maturation in vitro (Krisher \& Bavister 1998). Optimisation of in vitro maturation (IVM) protocols is vital not only for generating viable embryos but also to support the development of subsequent offspring into normal adults (Eppig \& O'Brien 1998).

Glucose, lactate, pyruvate and amino acids are prerequisites for the maturation and fertilisation of bovine oocytes in vitro. The oxidative metabolism of pyruvate and amino acids is the major energy-generating pathway in bovine oocytes (Rieger \& Loskutoff 1994). Furthermore, pyruvate and glucose support high levels of maturation, and amino acids aid pronuclear formation in fertilised oocytes (Donahue \& Stern 1968, Lim et al. 1999). Nutrient concentrations are non-physiological in mammalian IVM systems (Nakazawa et al. 1997); improvements in oocyte energy metabolism and developmental potential may be achieved by modelling nutrient profiles on those of preovulatory follicular fluid (FF) (Krisher \& Bavister 1998, Smitz \& Cortvrindt 1999).

FF has a variety of oocyte-related functions: maintenance of meiotic arrest (McNatty 1978), protection against proteolysis, extrusion during ovulation (Espey \& Lipner 
1994), enhancement of spermatozoa attraction, motility and acrosome reaction (Dell'Aquila et al. 1997, Rodriguez et al. 2001, Wang et al. 2001), and buffering against adverse haematic influences (Gosden et al. 1988). The components of FF are derived from plasma in early antral follicles (Gosden et al. 1988). In particular, antral lactate and pyruvate concentrations appear to result from granulosa cell glycolytic metabolism (Donahue \& Stern 1968, Gull et al. 1999).

Although undiluted FF is largely ineffective at supporting bovine oocyte maturation in vitro, it has been used to supplement media with varying degrees of success in equine, bovine and porcine systems, where oocyte maturation rates are affected by oestrous stage and follicle size (Gerritse et al. 1988, Sirard \& First 1988, Ayoub \& Hunter 1993, Choi et al. 1998, Takagi et al. 1998, Coy et al. 1999, Aguilar et al. 2001, Bøgh et al. 2002, Avery et al. 2003). This strategy exposes oocyte-cumulus complexes (OCCs) to a more 'physiological' - albeit undefined hormonal and nutritional milieu. By contrast, undefined/defined mammalian oocyte maturation systems - despite endocrine profiles conducive to cytoplasmic and nuclear maturation - contain a nutrient profile optimised for the culture of somatic cells rather than the combination of oocyte and cumulus cells (Zuelke \& Brackett 1993, Carolan et al. 1996, Eppig et al. 1996, Lebedeva et al. 1998, Smitz \& Cortvrindt 1999, Watson et al. 2000, Nagai 2001).

Some progress has been made towards the characterisation of FF in a variety of mammalian species. Glucose, pyruvate, lactate, histidine, phenylalanine and asparagine concentrations have been determined in human FF (Leese \& Lenton 1990, Jimena et al. 1993, Gull et al. 1999). Similarly, Guérin et al. (1995) determined amino acid profiles in FF from a variety of domestic species, albeit without consideration for follicle size or oestrous stage. Gérard et al. (2002) also quantified alanine, glutamate and glutamine profiles in equine FF.

The aim of the present study was to determine the carbohydrate and amino acid profile of bovine FF according to follicle dominance and oestrous stage. The data were compared with the nutrient composition of bovine plasma to help elucidate the role of granulosa cells in FF formation. Finally, dominant preovulatory FF composition (representing the in vivo environment) was compared with that of maturation medium (the in vitro environment). These data may contribute to the formulation of a nutritionally more 'physiological' bovine oocyte maturation medium.

\section{Materials and Methods Collection of FF and plasma}

Entire bovine reproductive tracts at different stages of the oestrous cycle were collected on ice and processed on site at a local abattoir within a few minutes of death to minimise post mortem changes in nutrient concentrations. Cycle stage (I-IV) was assessed on the basis of ovarian luteal morphology, according to the criteria defined by Ireland et al. (1980), on both ovaries from the same female. Briefly, Stage I (days 1-4) was defined as the interval between ovulation and the time when the epithelium grows over the rupture point, thus forming the apex of a new corpus luteum (CL). Stage II (days 5-10) covered the complete formation of a new CL with vasculature at its periphery; when bisected, the apex was red/brown while the remainder was orange/yellow. Stage III (days 11-17) was characterised by an absence of red/brown colouration, leaving the $\mathrm{CL}$ wholly orange/yellow, with visible apical vasculature later in this stage. During Stage IV (days 18-20), the ovary contained at least one large follicle and a regressed CL with no surface vasculature. Tracts with ovaries not clearly matching these criteria were discarded. Individual follicles were graded on the basis of dominance (assessed as whether they were the largest follicle or not, since steroid profiles were not determined) and FF volume. Mean (range) follicle diameter for Stages I-IV were $7.49 \pm 0.489,10.48 \pm 1.17$, $9.12 \pm 0.26$ and $11.52 \pm 6.07$ (6.76-9.63, 9.63-30.63, 7.65-11.38 and 4.50-33.25) $\mathrm{mm}$ for dominant follicles, and $5.30 \pm 0.30,7.18 \pm 0.31,6.46 \pm 0.26$ and $7.86 \pm 0.68$ (4.38-6.02, 7.00-10.50, 2.95-5.15 and 7.03-11.25) $\mathrm{mm}$ for non-dominant follicles respectively. Fluid was collected in 2 or $5 \mathrm{ml}$ sterile syringes (Becton Dickinson, Plymouth, Devon, UK) fitted with 19 gauge needles (Becton Dickinson). Cell debris was removed by centrifugation at $900 \mathrm{~g}$ for $5 \mathrm{~min}$ using a microcentrifuge (Micro Centaur; MSE Scientific, Loughborough, Leics, UK). The supernatant was diluted with HPLC grade water (Fisher Scientific, Loughborough, Leics, UK) for amino acid, glucose and lactate analyses, whilst undiluted fluid was used for determining pyruvate concentration.

Carotid blood was collected in $10 \mathrm{ml}$ EDTA tubes (NHS Logistics, Alfreton, Derbyshire, UK) from heifers immediately after slaughter. Blood samples were centrifuged at $250 \mathrm{~g}$ for $10 \mathrm{~min}$, and the supernatant plasma was processed as described above. All samples were processed and frozen on dry ice within 10-15 min of slaughter and stored at $-80^{\circ} \mathrm{C}$ until analysis.

\section{Composition of bovine maturation medium}

The bovine oocyte maturation medium used was based on bicarbonate-buffered TCM-199 supplemented with fetal calf serum (Sigma, Poole, Dorset, UK), as used routinely in our laboratories (original protocol from C Galli \& G Lazzari; Laboratorio di Tecnologie della Riproduzione (LTR-CIZ), Via Porcellasco 7/f, 26100 Cremona, Italy) (Gopichandran \& Leese 2003, Orsi \& Leese 2004a,b). Details of its supplements are given in Table 1. Four independent batches of maturation medium (using the same batch of serum) were tested, and samples were processed as described above. 
Table 1 Supplements for bovine maturation medium ( ${ }^{\mathrm{a}}$ Ferring Pharmaceuticals, Berks, Slough, UK).

\begin{tabular}{ll}
\hline Component & \multicolumn{1}{c}{ Concentration } \\
\hline Oestradiol-17 & $2 \mu \mathrm{g} / \mathrm{ml}$ \\
Apo-transferrin & $25 \mu \mathrm{g} / \mathrm{ml}$ \\
$\beta$-Mercaptoethanol & $7 \mathrm{nl} / \mathrm{ml}(97 \mu \mathrm{mol} / \mathrm{l})$ \\
Epidermal growth factor & $0.47 \mu \mathrm{gl} /$ \\
Fibroblast growth factor & $10.9 \mathrm{ng} / \mathrm{ml}$ \\
FSH/LH $^{\mathrm{a}}$ & $0.025 \mathrm{IU} / \mathrm{ml}$ of each \\
Fetal bovine serum & $10 \%(\mathrm{v} / \mathrm{v})$ \\
\hline
\end{tabular}

\section{Measurement of carbohydrate concentrations}

Glucose, pyruvate and lactate concentrations in FF $(n=121)$, plasma $(n=7)$ and maturation medium $(n=4)$ were measured enzymatically on a COBAS MIRA autoanalyser (Roche Instruments, Lewes, East Sussex, UK), as previously described (Leese \& Lenton 1990).

\section{Measurement of amino acid profiles}

Amino acid concentrations were measured by reversephase HPLC, as previously described (Partridge \& Leese 1996). Sample profiles ( $n=10 \mathrm{FFs}$ for each oestrous stage; $n=7$ for plasma; $n=4$ for maturation medium) were compared with $10 \mu \mathrm{M}$ amino acid standards. All were analysed on a Kontron 500 Series automated HPLC system fitted with a Jasco F920 fluorescence detector and $4.5 \times 250 \mathrm{~mm}$ Hypersil ODS-16 column (Jones Chromatography, Mid Glamorgan, Pontypridd, UK). Tetrahydrofuran concentration was modified over the $7-12 \%$ range according to the elution profile. All samples were analysed at two different dilutions to allow for the elevated concentrations of glycine, glutamine and alanine. This method did not allow the detection of proline or cysteine.

\section{Measurement of osmolality}

Osmolality was measured using pooled FF, independent of oestrous stage. Samples $(100 \mu \mathrm{l})$ were measured in triplicate on an osmometer (Camlab, Over, Cambs, UK).

\section{Data presentation and statistical analysis}

Data are presented as means \pm S.E.M. and tested for normality using Anderson-Darling tests. Significant differences in FF nutrient profile across the oestrous cycle and comparisons with plasma were tested by one-way ANOVA, followed by Fisher's LSD test post hoc, or by Kruskal-Wallis test followed by Mann-Whitney $U$ tests post hoc. Differences between dominant and nondominant follicle parameters were compared by Student's t-test or Mann-Whitney $U$ test, as were the differences between the nutrient composition of preovulatory FF and IVM medium.

\section{Results}

\section{Carbohydrate concentrations of FF and plasma}

There were no significant differences in pyruvate concentrations according to oestrous cycle stage or between plasma and FF in dominant follicles (Table 2). By contrast, FF glucose was slightly, but significantly, lower than that of plasma in all instances (except Stage II), while the opposite trend was observed for lactate, particularly Stage I follicles $(P<0.05)$.

Non-dominant FF pyruvate levels were similar across oestrous cycle stages and in plasma, while glucose concentration was significantly lower than that of plasma in all cases (except Stage II) $(P<0.05)$ (Table 3). Lactate concentration was higher in FF than plasma, particularly in Stage I follicles where levels were significantly higher than at other stages $(P<0.05)$.

There was no significant difference in pyruvate and glucose concentrations on the basis of follicle dominance throughout the oestrous cycle (Table 3). However, Stage III and IV lactate profiles were higher in non-dominant follicles compared with their stage-matched dominant counterparts $(P<0.01)$. Independent of dominance, glucose and lactate levels were lower and higher respectively in Stage I follicles.

\section{Amino acid profiles of FF and plasma}

Most amino acid concentrations were affected by oestrous stage, although this was largely attributable to differences between dominant Stage I FF and the other stages, where amino acid concentrations were consistently

Table 2 Carbohydrate concentrations of dominant bovine ovarian follicles throughout the oestrous cycle and compared with plasma (comparisons are confined to within columns).

\begin{tabular}{lccc}
\hline & Pyruvate $(\mathrm{mmol} / \mathrm{l})$ & Glucose $(\mathrm{mmol} / \mathrm{l})$ & Lactate $(\mathrm{mmol} / \mathrm{l})$ \\
\hline Stage I & $0.01 \pm 0.01$ & $4.71 \pm 0.31^{\mathrm{a}}$ & $8.16 \pm 1.60^{\mathrm{a}}$ \\
Stage II & $0.04 \pm 0.01$ & $5.08 \pm 0.20^{\mathrm{ab}}$ & $5.01 \pm 0.39^{\mathrm{b}}$ \\
Stage III & $0.05 \pm 0.01$ & $4.99 \pm 0.09^{\mathrm{a}}$ & $5.22 \pm 0.26^{\mathrm{b}}$ \\
Stage IV & $0.03 \pm 0.01$ & $4.84 \pm 0.14^{\mathrm{a}}$ & $5.09 \pm 0.37^{\mathrm{b}}$ \\
Plasma & $0.01 \pm 0.01$ & $5.57 \pm 0.14^{\mathrm{b}}$ & $3.94 \pm 0.35^{\mathrm{c}}$ \\
\hline
\end{tabular}

Means without common superscripts differ $(P<0.05)$.

Table 3 Pyruvate, glucose and lactate concentrations of nondominant bovine ovarian follicles throughout the oestrous cycle compared with plasma.

\begin{tabular}{lccc}
\hline & Pyruvate $(\mathrm{mmol} / \mathrm{l})$ & Glucose $(\mathrm{mmol} / \mathrm{l})$ & Lactate $(\mathrm{mmol} / \mathrm{l})$ \\
\hline Stage I & $0.00 \pm 0.00$ & $4.25 \pm 0.35^{\mathrm{a}}$ & $10.16 \pm 1.60^{\mathrm{a}}$ \\
Stage II & $0.02 \pm 0.01$ & $5.05 \pm 0.24^{\mathrm{ab}}$ & $7.39 \pm 0.72^{\mathrm{b}}$ \\
Stage III & $0.03 \pm 0.01$ & $4.49 \pm 0.12^{\mathrm{a}}$ & $8.51 \pm 2.01^{\mathrm{b}, * *}$ \\
Stage IV & $0.03 \pm 0.01$ & $4.54 \pm 0.26^{\mathrm{a}}$ & $5.65 \pm 0.11^{\mathrm{b}, * *}$ \\
Plasma & $0.01 \pm 0.01$ & $5.57 \pm 0.14^{\mathrm{b}}$ & $3.94 \pm 0.35^{\mathrm{c}}$ \\
\hline
\end{tabular}

Means without common superscripts differ $(P<0.05)$. ${ }^{* *} P<0.01$ in lactate profile in stage-matched dominant versus non-dominant FF. 
lower (Table 4). Amino acids affected by oestrous stage included: glutamate, asparagine, histidine, glutamine, threonine, arginine, taurine, alanine, tyrosine, tryptophan, methionine, valine, phenylalanine, leucine and lysine. The Stage I FF amino acid profile was very similar to that of plasma, particularly as regards aspartate, glutamate, serine, glycine, threonine, arginine, taurine, phenylalanine and isoleucine. By contrast, Stages II-IV profiles were significantly lower than plasma for aspartate, glutamate, asparagine (Stage III only), serine, taurine (except Stage II), alanine (Stage II only), methionine (except Stage II), valine (except Stage II), leucine (Stage III only) and lysine (except Stage III). Overall, plasma levels were markedly higher than those of FF for glutamate, serine and glutamine and, to a lesser extent, for asparagine, threonine, taurine, tyrosine, methionine, phenylalanine and lysine. By contrast, FF valine concentration was significantly higher than in plasma.

Alterations in amino acid profile with respect to oestrous stage and plasma were less evident for non-dominant follicles (Table 5). Only glutamate, alanine, leucine and lysine were affected by oestrous stage. Compared with plasma, FF had lower aspartate, glutamate (except Stage I) and serine (except Stage I), while glutamine (except Stages I and II), alanine (only Stage I) and lysine (except Stage II) levels were higher.

Table 4 Bovine FF amino acid profile of dominant follicles at different stages of oestrus compared with plasma.

\begin{tabular}{|c|c|c|c|c|c|}
\hline Amino acid & Stage I $(\mathrm{mmol} / \mathrm{l})$ & Stage II $(\mathrm{mmol} / \mathrm{l})$ & Stage III (mmol/l) & Stage IV $(\mathrm{mmol} / \mathrm{l})$ & Plasma $(\mathrm{mmol} / \mathrm{l})$ \\
\hline ASP & $0.03 \pm 0.01^{\mathrm{ab}}$ & $0.02 \pm 0.00^{b}$ & $0.01 \pm 0.00^{b}$ & $0.012 \pm 0.002^{b}$ & $0.055 \pm 0.014^{\mathrm{a}}$ \\
\hline GLU & $0.18 \pm 0.01^{\mathrm{a}}$ & $0.07 \pm 0.03^{b}$ & $0.05 \pm 0.01^{b}$ & $0.06 \pm 0.01^{b}$ & $0.20 \pm 0.02^{\mathrm{a}}$ \\
\hline ASN & $0.06 \pm 0.01^{\mathrm{a}}$ & $0.03 \pm 0.00^{\mathrm{bc}}$ & $0.02 \pm 0.00^{\mathrm{C}}$ & $0.03 \pm 0.00^{b c}$ & $0.04 \pm 0.01^{b}$ \\
\hline SER & $0.14 \pm 0.00^{\mathrm{ab}}$ & $0.07 \pm 0.01^{b}$ & $0.05 \pm 0.01^{b}$ & $0.06 \pm 0.01^{b}$ & $0.21 \pm 0.07^{\mathrm{a}}$ \\
\hline HIS & $0.15 \pm 0.00^{\mathrm{a}}$ & $0.09 \pm 0.02^{b}$ & $0.06 \pm 0.00^{b}$ & $0.08 \pm 0.01^{b}$ & $0.06 \pm 0.02^{b}$ \\
\hline GLN & $0.88 \pm 0.12^{\mathrm{a}}$ & $0.44 \pm 0.04^{\mathrm{b}}$ & $0.34 \pm 0.03^{b}$ & $0.35 \pm 0.04^{b}$ & $0.32 \pm 0.03^{b}$ \\
\hline GLY & $0.87 \pm 0.18$ & $0.55 \pm 0.05$ & $0.42 \pm 0.05$ & $0.49 \pm 0.05$ & $0.51 \pm 0.10$ \\
\hline THR & $0.37 \pm 0.03^{\mathrm{a}}$ & $0.26 \pm 0.02^{\mathrm{abc}}$ & $0.21 \pm 0.03^{b c}$ & $0.22 \pm 0.02^{\mathrm{c}}$ & $0.31 \pm 0.05^{\mathrm{ac}}$ \\
\hline ARG & $0.22 \pm 0.01^{\mathrm{a}}$ & $0.21 \pm 0.01^{\mathrm{ab}}$ & $0.13 \pm 0.01^{b}$ & $0.14 \pm 0.01^{\mathrm{ab}}$ & $0.18 \pm 0.04^{\mathrm{ab}}$ \\
\hline TAU & $0.14 \pm 0.02^{\mathrm{a}}$ & $0.09 \pm 0.01^{\mathrm{ab}}$ & $0.08 \pm 0.01^{b}$ & $0.08 \pm 0.01^{b}$ & $0.12 \pm 0.02^{\mathrm{a}}$ \\
\hline ALA & $0.68 \pm 0.04^{\mathrm{a}}$ & $0.54 \pm 0.06^{b}$ & $0.38 \pm 0.03^{\mathrm{c}}$ & $0.46 \pm 0.02^{b c}$ & $0.41 \pm 0.04^{\mathrm{C}}$ \\
\hline TYR & $0.13 \pm 0.01^{a}$ & $0.09 \pm 0.01^{b}$ & $0.06 \pm 0.01^{b}$ & $0.08 \pm 0.01^{\mathrm{b}}$ & $0.10 \pm 0.01^{b}$ \\
\hline TRP & $0.12 \pm 0.01^{\mathrm{a}}$ & $0.06 \pm 0.00^{b}$ & $0.05 \pm 0.01^{b}$ & $0.06 \pm 0.01^{b}$ & $0.04 \pm 0.01^{b}$ \\
\hline MET & $0.06 \pm 0.00^{\mathrm{a}}$ & $0.05 \pm 0.00^{b c}$ & $0.03 \pm 0.01^{b}$ & $0.04 \pm 0.01^{b}$ & $0.04 \pm 0.01^{\mathrm{c}}$ \\
\hline VAL & $0.53 \pm 0.03^{\mathrm{a}}$ & $0.36 \pm 0.01^{b c}$ & $0.30 \pm 0.03^{b}$ & $0.35 \pm 0.03^{b}$ & $0.04 \pm 0.02^{\mathrm{c}}$ \\
\hline PHE & $0.13 \pm 0.01^{\mathrm{a}}$ & $0.10 \pm 0.01^{b c}$ & $0.08 \pm 0.01^{b}$ & $0.08 \pm 0.01^{\mathrm{b}}$ & $0.11 \pm 0.01^{\mathrm{ac}}$ \\
\hline ILE & $0.20 \pm 0.02$ & $0.16 \pm 0.01$ & $0.14 \pm 0.01$ & $0.16 \pm 0.01$ & $0.17 \pm 0.11$ \\
\hline LEU & $0.33 \pm 0.03^{a}$ & $0.24 \pm 0.01^{\mathrm{bc}}$ & $0.19 \pm 0.02^{b}$ & $0.21 \pm 0.02^{b c}$ & $0.26 \pm 0.01^{c}$ \\
\hline LYS & $0.21 \pm 0.01^{a}$ & $0.20 \pm 0.03^{\mathrm{a}}$ & $0.11 \pm 0.01^{b}$ & $0.17 \pm 0.02^{\mathrm{a}}$ & $0.10 \pm 0.01^{b}$ \\
\hline
\end{tabular}

Means without common superscripts $\operatorname{differ}(P<0.05)$.

Table 5 Bovine FF amino acid profiles of non-dominant follicles at different stages of oestrus compared with plasma.

\begin{tabular}{|c|c|c|c|c|c|}
\hline Amino acid & Stage I $(\mathrm{mmol} / \mathrm{l})$ & Stage II $(\mathrm{mmol} / \mathrm{l})$ & Stage III $(\mathrm{mmol} / \mathrm{l})$ & Stage IV $(\mathrm{mmol} / \mathrm{l})$ & Plasma $(\mathrm{mmol} / \mathrm{l})$ \\
\hline$A S P$ & $0.03 \pm 0.01^{\mathrm{a}}$ & $0.01 \pm 0.00^{\mathrm{a}}$ & $0.02 \pm 0.00^{a_{,} *}$ & $0.02 \pm 0.00^{\mathrm{a}}$ & $0.06 \pm 0.01^{\mathrm{b}}$ \\
\hline GLU & $0.20 \pm 0.04^{\mathrm{ac}}$ & $0.09 \pm 0.02^{b}$ & $0.13 \pm 0.03^{\mathrm{ab}, *}$ & $0.13 \pm 0.03^{\mathrm{ab}}$ & $0.20 \pm 0.02^{c}$ \\
\hline ASN & $0.03 \pm 0.01$ & $0.03 \pm 0.01$ & $0.03 \pm 0.01$ & $0.03 \pm 0.00$ & $0.04 \pm 0.01$ \\
\hline SER & $0.09 \pm 0.02^{\mathrm{ab}}$ & $0.05 \pm 0.01^{\mathrm{a}}$ & $0.07 \pm 0.01^{\mathrm{a}}$ & $0.07 \pm 0.01^{\mathrm{a}}$ & $0.21 \pm 0.07^{b}$ \\
\hline HIS & $0.10 \pm 0.01$ & $0.07 \pm 0.01$ & $0.08 \pm 0.01 *$ & $0.09 \pm 0.12$ & $0.06 \pm 0.02$ \\
\hline GLN & $0.51 \pm 0.09^{\mathrm{ab}}$ & $0.34 \pm 0.09^{\mathrm{ab}}$ & $0.53 \pm 0.10^{\mathrm{a}}$ & $0.54 \pm 0.04^{\mathrm{a}, *}$ & $0.32 \pm 0.03^{b}$ \\
\hline GLY & $0.86 \pm 0.15$ & $0.38 \pm 0.07$ & $0.56 \pm 0.05$ & $0.63 \pm 0.07$ & $0.51 \pm 0.10$ \\
\hline THR & $0.26 \pm 0.05$ & $0.21 \pm 0.03$ & $0.26 \pm 0.04$ & $0.29 \pm 0.02 *$ & $0.31 \pm 0.05$ \\
\hline ARG & $0.21 \pm 0.01$ & $0.15 \pm 0.02$ & $0.18 \pm 0.01^{*}$ & $0.19 \pm 0.01^{*}$ & $0.18 \pm 0.04$ \\
\hline TAU & $0.15 \pm 0.04$ & $0.07 \pm 0.01$ & $0.13 \pm 0.03$ & $0.13 \pm 0.02$ & $0.12 \pm 0.02$ \\
\hline$A L A$ & $0.81 \pm 0.10^{\mathrm{a}}$ & $0.48 \pm 0.05^{b}$ & $0.55 \pm 0.08^{b}$ & $0.51 \pm 0.02^{b}$ & $0.41 \pm 0.04^{b}$ \\
\hline TYR & $0.11 \pm 0.01$ & $0.07 \pm 0.02$ & $0.09 \pm 0.01 *$ & $0.11 \pm 0.01^{*}$ & $0.10 \pm 0.01$ \\
\hline TRP & $0.08 \pm 0.02$ & $0.05 \pm 0.01$ & $0.07 \pm 0.01$ & $0.07 \pm 0.00$ & $0.04 \pm 0.01$ \\
\hline MET & $0.05 \pm 0.00$ & $0.03 \pm 0.01$ & $0.05 \pm 0.01 *$ & $0.05 \pm 0.00^{*}$ & $0.04 \pm 0.01$ \\
\hline VAL & $0.42 \pm 0.01$ & $0.27 \pm 0.06$ & $0.37 \pm 0.04$ & $0.41 \pm 0.01$ & $0.43 \pm 0.02$ \\
\hline PHE & $0.13 \pm 0.01$ & $0.08 \pm 0.01$ & $0.11 \pm 0.01^{*}$ & $0.10 \pm 0.01^{*}$ & $0.11 \pm 0.01$ \\
\hline ILE & $0.18 \pm 0.01$ & $0.15 \pm 0.03$ & $0.16 \pm 0.03$ & $0.20 \pm 0.01^{*}$ & $0.17 \pm 0.01$ \\
\hline$L E U$ & $0.32 \pm 0.03^{\mathrm{ac}}$ & $0.20 \pm 0.04^{b}$ & $0.27 \pm 0.02^{\mathrm{ac}}$ & $0.30 \pm 0.01^{\mathrm{c}, * *}$ & $0.26 \pm 0.01^{\mathrm{ac}}$ \\
\hline$L Y S$ & $0.22 \pm 0.03^{\mathrm{ac}}$ & $0.13 \pm 0.04^{\mathrm{ab}}$ & $0.16 \pm 0.02^{\mathrm{ac}, *}$ & $0.22 \pm 0.03^{c}$ & $0.10 \pm 0.01^{\mathrm{b}}$ \\
\hline
\end{tabular}

Different superscripts indicate significant differences in amino acid concentration between groups $(P<0.05) .{ }^{*} P<0.05,{ }^{* *} P<0.01$ statistically significant differences in amino acid profile between stage-matched dominant versus non-dominant FF. 
There were no significant differences in amino acid profile between dominant and non-dominant FF for the early oestrous stages, although asparagine and methionine profiles were noticeably lower in Stage I non-dominant FF, while taurine concentration was reduced in their Stage II counterparts (all $P=0.052$ ) (Table 5). By contrast, nondominant Stage III FF had significantly higher concentrations of aspartate, glutamate, histidine, arginine, tyrosine, methionine, phenylalanine and lysine. Similarly, Stage IV non-dominant FF was characterised by significantly higher levels of glutamine, threonine, arginine tyrosine, methionine, phenylalanine, isoleucine and leucine.

\section{Correlations between FF volume, carbohydrate and amino acid profiles}

Glucose concentration was not significantly correlated with FF volume (although a trend was observed), while pyruvate and lactate concentrations were related to FF volume positively and negatively respectively (Table 6). Pyruvate concentration was not correlated with that of the other carbohydrates, whereas glucose and lactate concentrations were significantly negatively correlated.

Almost all amino acids were significantly positively correlated with each other (Table 7 ). Correlations were weakest between amino acids and FF volume. Aspartate, glutamate, alanine, tyrosine, methionine, phenylalanine and leucine were all significantly negatively correlated with FF volume and arginine weakly so.

There was no significant correlation between pyruvate profile and that of any of the amino acids (Table 8). However, there was a trend for a negative correlation between glucose and amino acid profiles, which was significant for arginine, tyrosine, methionine, phenylalanine, isoleucine, leucine and lysine $(P<0.05)$. In contrast, there was a trend for a positive correlation between lactate and amino acid profiles, which was significant for aspartate, glutamate, alanine, tyrosine and phenylalanine $(P<0.05)$, and approached significance for arginine.

\section{Comparison of FF with maturation medium}

There was a significantly higher pyruvate concentration in the medium used for in vitro maturation compared with Stage IV FF, whereas the reverse was true for lactate $(P<0.001)$ (Table 9). Glucose concentration was similar in maturation medium and Stage IV FF.

Table 6 Bovine FF volume and carbohydrate correlation coefficients; $r$ and $P$ values.

\begin{tabular}{|c|c|c|c|}
\hline & Volume & Pyruvate & Glucose \\
\hline Pyruvate & $0.346 ; 0.022$ & - & - \\
\hline Glucose & $0.271 ; 0.075$ & $-0.001 ; 0.997$ & - \\
\hline Lactate & $-0.326 ; 0.031$ & $0.139 ; 0.367$ & $-0.309 ; 0.041$ \\
\hline
\end{tabular}

Bold indicates significance $(P<0.05)$.
All amino acid concentrations (Fig. 1) differed significantly between maturation medium and preovulatory FF, except those of histidine, taurine, alanine and tryptophan. Apart from the elevated concentrations of aspartate, glutamate and serine in maturation medium, the overall amino acid ratios were similar in both groups, with FF concentrations being consistently lower.

The osmolality of FF $(292.4 \pm 2.1 \mathrm{mOsm} / \mathrm{kg})$ was not significantly different from that of maturation medium $(284.5 \pm 4.9 \mathrm{mOsm} / \mathrm{kg})$ and plasma (299.0 \pm $5.0 \mathrm{mOsm} / \mathrm{kg})$.

\section{Discussion \\ Carbohydrate profiles}

We have measured the pyruvate, glucose, lactate and amino acid composition of bovine FF throughout the oestrous cycle. FF pyruvate concentration was largely unaffected by oestrous stage and follicle dominance. Apart from Stage IV dominant FF, it increased moderately in line with oestrous stage, possibly as a result of increased production by granulosa/cumulus cell activity (Leese \& Barton 1985). By contrast, glucose concentration was relatively uniform throughout the oestrous cycle and independent of follicle dominance, with lowest values reported for Stage I. These follicles were, on average, smaller than their later stage counterparts and, in this respect, Leese \& Lenton (1990) also found that concentrations of glucose were affected by follicle volume in the human. However, the present study reports significantly higher lactate concentrations in Stage I follicles, independent of dominance, a trend which agrees with a previous report (Leroy et al. 2004). The glucose and lactate levels may be accounted for by (i) the elevated metabolic activity of small follicles (Hammon et al. 2000a) and/or (ii) the possible atretic fate of a significant number of the antral follicles sampled (Erickson 1966, Choudary et al. 1968, Kruip \& Dieleman 1982, Wise 1987). The trend for lactate concentration to be higher in non-dominant follicles (significant for later oestrous stages) supports the second explanation.

The plasma glucose concentrations reported are at the upper end of the physiological range for cattle (40$100 \mathrm{mg} / \mathrm{dl}$; lowa State University Clinical Pathology Laboratory) and the FF concentrations are higher than those quoted in a recent study (Leroy et al. 2004). We therefore repeated these analyses (and those of fresh follicles obtained from a different slaughterhouse for comparison) using undiluted samples with an alternative assay (Glucose PAP; Audit Diagnostics, Doughcloyne, Cork, Ireland) on a different autoanalyser (COBAS MIRA S Plus; Roche Instruments, UK). Our measurements were reproducible; these discrepancies may be a reflection of stress associated with progress along the abattoir line and/or, possibly, to post mortem changes in the study of Leroy et al. (2004). Plasma lactate levels were also mildly elevated, whereas 
Table 7 Correlation coefficients between bovine FF volume and amino acid concentrations: $r$ and $P$ values.

\begin{tabular}{|c|c|c|c|c|c|c|c|c|c|c|c|c|c|c|c|c|c|c|c|}
\hline & Volume & ASP & GLU & ASN & SER & HIS & GLN & GLY & THR & ARG & TAU & ALA & TYR & TRP & MET & VAL & PHE & ILE & LEU \\
\hline ASP & $\begin{array}{r}-0.372 \\
0.013\end{array}$ & - & - & - & - & - & - & - & - & - & - & - & - & - & - & & & - & - \\
\hline GLU & $\begin{array}{r}-0.397 \\
\mathbf{0 . 0 0 8}\end{array}$ & $\begin{array}{l}0.703 \\
0.000\end{array}$ & - & - & - & - & - & - & - & - & - & - & - & - & - & - & - & - & - \\
\hline ASN & $\begin{array}{r}-0.218 \\
0.156\end{array}$ & $\begin{array}{l}0.296 \\
0.051\end{array}$ & $\begin{array}{l}0.331 \\
0.028\end{array}$ & - & - & - & - & - & - & - & - & - & - & - & - & - & - & - & - \\
\hline SER & $\begin{array}{r}-0.165 \\
0.283\end{array}$ & $\begin{array}{l}0.589 \\
0.000\end{array}$ & $\begin{array}{l}0.413 \\
0.005\end{array}$ & $\begin{array}{l}0.555 \\
0.000\end{array}$ & - & - & - & - & - & - & - & - & - & - & - & - & - & - & - \\
\hline HIS & $\begin{array}{r}-0.204 \\
0.185\end{array}$ & $\begin{array}{l}0.432 \\
0.003\end{array}$ & $\begin{array}{l}0.346 \\
0.021\end{array}$ & $\begin{array}{l}0.727 \\
0.000\end{array}$ & $\begin{array}{l}0.533 \\
0.000\end{array}$ & - & - & - & - & - & - & - & - & - & - & - & - & - & - \\
\hline GLN & $\begin{array}{r}-0.256 \\
0.094\end{array}$ & $\begin{array}{l}0.277 \\
0.069\end{array}$ & $\begin{array}{l}0.281 \\
0.065\end{array}$ & $\begin{array}{l}0.812 \\
0.000\end{array}$ & $\begin{array}{l}0.587 \\
0.000\end{array}$ & $\begin{array}{l}0.677 \\
0.000\end{array}$ & - & - & - & - & - & - & - & - & - & - & - & - & - \\
\hline GLY & $\begin{array}{r}-0.230 \\
0.139\end{array}$ & $\begin{array}{l}0.554 \\
0.000\end{array}$ & $\begin{array}{l}0.522 \\
0.000\end{array}$ & $\begin{array}{l}0.527 \\
0.000\end{array}$ & $\begin{array}{l}0.708 \\
0.000\end{array}$ & $\begin{array}{l}0.651 \\
0.000\end{array}$ & $\begin{array}{l}0.570 \\
0.000\end{array}$ & - & - & - & - & - & - & - & - & - & - & - & - \\
\hline THR & $\begin{array}{r}-0.108 \\
0.486\end{array}$ & $\begin{array}{l}0.296 \\
0.051\end{array}$ & $\begin{array}{l}0.300 \\
0.048\end{array}$ & $\begin{array}{l}0.716 \\
0.000\end{array}$ & $\begin{array}{l}0.529 \\
0.000\end{array}$ & $\begin{array}{l}0.597 \\
0.000\end{array}$ & $\begin{array}{l}0.688 \\
0.000\end{array}$ & $\begin{array}{l}0.495 \\
0.001\end{array}$ & - & - & - & - & - & - & - & - & - & - & - \\
\hline ARG & $\begin{array}{r}-0.288 \\
0.058\end{array}$ & $\begin{array}{l}0.551 \\
0.000\end{array}$ & $\begin{array}{l}0.471 \\
0.001\end{array}$ & $\begin{array}{l}0.714 \\
0.000\end{array}$ & $\begin{array}{l}0.630 \\
0.000\end{array}$ & $\begin{array}{l}0.645 \\
0.000\end{array}$ & $\begin{array}{l}0.726 \\
0.000\end{array}$ & $\begin{array}{l}0.696 \\
0.000\end{array}$ & $\begin{array}{l}0.681 \\
0.000\end{array}$ & - & - & - & - & - & - & - & - & - & - \\
\hline TAU & $\begin{array}{r}-0.169 \\
0.272\end{array}$ & $\begin{array}{l}0.332 \\
0.028\end{array}$ & $\begin{array}{l}0.440 \\
0.003\end{array}$ & $\begin{array}{l}0.436 \\
0.003\end{array}$ & $\begin{array}{l}0.423 \\
0.004\end{array}$ & $\begin{array}{l}0.349 \\
0.020\end{array}$ & $\begin{array}{l}0.604 \\
0.000\end{array}$ & $\begin{array}{l}0.340 \\
0.025\end{array}$ & $\begin{array}{l}0.581 \\
0.000\end{array}$ & $\begin{array}{l}0.435 \\
0.003\end{array}$ & - & - & - & - & - & - & - & - & - \\
\hline ALA & $\begin{array}{r}-0.297 \\
0.050\end{array}$ & $\begin{array}{l}0.508 \\
0.000\end{array}$ & $\begin{array}{l}0.683 \\
0.000\end{array}$ & $\begin{array}{l}0.566 \\
0.003\end{array}$ & $\begin{array}{l}0.459 \\
0.002\end{array}$ & $\begin{array}{l}0.586 \\
0.000\end{array}$ & $\begin{array}{l}0.475 \\
0.001\end{array}$ & $\begin{array}{l}0.566 \\
0.000\end{array}$ & $\begin{array}{l}0.401 \\
0.007\end{array}$ & $\begin{array}{l}0.630 \\
0.000\end{array}$ & $\begin{array}{l}0.534 \\
0.000\end{array}$ & - & - & - & - & - & - & - & - \\
\hline TYR & $\begin{array}{r}-0.353 \\
0.019\end{array}$ & $\begin{array}{l}0.566 \\
0.000\end{array}$ & $\begin{array}{l}0.500 \\
0.001\end{array}$ & $\begin{array}{l}0.776 \\
0.000\end{array}$ & $\begin{array}{l}0.582 \\
0.000\end{array}$ & $\begin{array}{l}0.750 \\
0.000\end{array}$ & $\begin{array}{l}0.669 \\
0.000\end{array}$ & $\begin{array}{l}0.665 \\
0.000\end{array}$ & $\begin{array}{l}0.651 \\
0.000\end{array}$ & $\begin{array}{l}0.809 \\
0.000\end{array}$ & $\begin{array}{l}0.401 \\
0.007\end{array}$ & $\begin{array}{l}0.646 \\
0.000\end{array}$ & - & - & - & - & - & - & - \\
\hline TRP & $\begin{array}{r}-0.233 \\
0.128\end{array}$ & $\begin{array}{l}0.242 \\
0.113\end{array}$ & $\begin{array}{l}0.372 \\
0.013\end{array}$ & $\begin{array}{l}0.719 \\
0.000\end{array}$ & $\begin{array}{l}0.591 \\
0.000\end{array}$ & $\begin{array}{l}0.658 \\
0.000\end{array}$ & $\begin{array}{l}0.725 \\
0.000\end{array}$ & $\begin{array}{l}0.535 \\
0.000\end{array}$ & $\begin{array}{l}0.623 \\
0.000\end{array}$ & $\begin{array}{l}0.487 \\
0.001\end{array}$ & $\begin{array}{l}0.607 \\
0.000\end{array}$ & $\begin{array}{l}0.581 \\
0.000\end{array}$ & $\begin{array}{l}0.626 \\
0.000\end{array}$ & - & - & - & - & - & - \\
\hline MET & $\begin{array}{r}-0.338 \\
0.025\end{array}$ & $\begin{array}{l}0.428 \\
0.001\end{array}$ & $\begin{array}{l}0.379 \\
0.011\end{array}$ & $\begin{array}{l}0.785 \\
0.000\end{array}$ & $\begin{array}{l}0.572 \\
0.000\end{array}$ & $\begin{array}{l}0.652 \\
0.000\end{array}$ & $\begin{array}{l}0.749 \\
0.000\end{array}$ & $\begin{array}{l}0.580 \\
0.000\end{array}$ & $\begin{array}{l}0.636 \\
0.000\end{array}$ & $\begin{array}{l}0.855 \\
0.000\end{array}$ & $\begin{array}{l}0.511 \\
0.000\end{array}$ & $\begin{array}{l}0.612 \\
0.000\end{array}$ & $\begin{array}{l}0.901 \\
0.000\end{array}$ & $\begin{array}{l}0.656 \\
0.000\end{array}$ & - & - & - & - & - \\
\hline VAL & $\begin{array}{r}-0.239 \\
0.119\end{array}$ & $\begin{array}{l}0.295 \\
0.052\end{array}$ & $\begin{array}{l}0.360 \\
0.016\end{array}$ & $\begin{array}{l}0.754 \\
0.000\end{array}$ & $\begin{array}{l}0.588 \\
0.000\end{array}$ & $\begin{array}{l}0.679 \\
0.000\end{array}$ & $\begin{array}{l}0.698 \\
0.000\end{array}$ & $\begin{array}{l}0.595 \\
0.000\end{array}$ & $\begin{array}{l}0.848 \\
0.000\end{array}$ & $\begin{array}{l}0.677 \\
0.000\end{array}$ & $\begin{array}{l}0.510 \\
0.000\end{array}$ & $\begin{array}{l}0.515 \\
0.000\end{array}$ & $\begin{array}{l}0.741 \\
0.000\end{array}$ & $\begin{array}{l}0.767 \\
0.000\end{array}$ & $\begin{array}{l}0.722 \\
0.000\end{array}$ & - & - & - & - \\
\hline PHE & $\begin{array}{r}-0.461 \\
0.002\end{array}$ & $\begin{array}{l}0.536 \\
0.000\end{array}$ & $\begin{array}{l}0.559 \\
0.000\end{array}$ & $\begin{array}{l}0.647 \\
0.000\end{array}$ & $\begin{array}{l}0.528 \\
0.000\end{array}$ & $\begin{array}{l}0.681 \\
0.000\end{array}$ & $\begin{array}{l}0.630 \\
0.000\end{array}$ & $\begin{array}{l}0.677 \\
0.000\end{array}$ & $\begin{array}{l}0.504 \\
0.000\end{array}$ & $\begin{array}{l}0.765 \\
0.000\end{array}$ & $\begin{array}{l}0.405 \\
0.006\end{array}$ & $\begin{array}{l}0.708 \\
0.000\end{array}$ & $\begin{array}{l}0.888 \\
0.000\end{array}$ & $\begin{array}{l}0.633 \\
0.000\end{array}$ & $\begin{array}{l}0.864 \\
0.000\end{array}$ & $\begin{array}{l}0.716 \\
0.000\end{array}$ & - & - & - \\
\hline ILE & $\begin{array}{r}-0.196 \\
0.203\end{array}$ & $\begin{array}{l}0.375 \\
0.012\end{array}$ & $\begin{array}{l}0.320 \\
0.034\end{array}$ & $\begin{array}{l}0.663 \\
0.000\end{array}$ & $\begin{array}{l}0.529 \\
0.000\end{array}$ & $\begin{array}{l}0.540 \\
0.000\end{array}$ & $\begin{array}{l}0.559 \\
0.000\end{array}$ & $\begin{array}{l}0.490 \\
0.000\end{array}$ & $\begin{array}{l}0.496 \\
0.001\end{array}$ & $\begin{array}{l}0.676 \\
0.000\end{array}$ & $\begin{array}{l}0.376 \\
0.012\end{array}$ & $\begin{array}{l}0.492 \\
0.001\end{array}$ & $\begin{array}{l}0.742 \\
0.000\end{array}$ & $\begin{array}{l}0.524 \\
0.000\end{array}$ & $\begin{array}{l}0.716 \\
0.000\end{array}$ & $\begin{array}{l}0.651 \\
0.000\end{array}$ & $\begin{array}{l}0.661 \\
0.000\end{array}$ & - & - \\
\hline LEU & $\begin{array}{r}-0.337 \\
0.025\end{array}$ & $\begin{array}{l}0.480 \\
0.001\end{array}$ & $\begin{array}{l}0.446 \\
0.002\end{array}$ & $\begin{array}{l}0.586 \\
0.000\end{array}$ & $\begin{array}{l}0.632 \\
0.000\end{array}$ & $\begin{array}{l}0.553 \\
0.000\end{array}$ & $\begin{array}{l}0.588 \\
0.000\end{array}$ & $\begin{array}{l}0.691 \\
0.000\end{array}$ & $\begin{array}{l}0.704 \\
0.000\end{array}$ & $\begin{array}{l}0.746 \\
0.000\end{array}$ & $\begin{array}{l}0.485 \\
0.001\end{array}$ & $\begin{array}{l}0.544 \\
0.000\end{array}$ & $\begin{array}{l}0.789 \\
0.000\end{array}$ & $\begin{array}{l}0.628 \\
0.000\end{array}$ & $\begin{array}{l}0.771 \\
0.000\end{array}$ & $\begin{array}{l}0.842 \\
0.000\end{array}$ & $\begin{array}{l}0.814 \\
0.000\end{array}$ & $\begin{array}{l}0.745 \\
0.000\end{array}$ & - \\
\hline LYS & $\begin{array}{r}-0.213 \\
0.165\end{array}$ & $\begin{array}{l}0.410 \\
0.006\end{array}$ & $\begin{array}{l}0.254 \\
0.097\end{array}$ & $\begin{array}{l}0.618 \\
0.001\end{array}$ & $\begin{array}{l}0.382 \\
0.010\end{array}$ & $\begin{array}{l}0.695 \\
0.001\end{array}$ & $\begin{array}{l}0.455 \\
0.002\end{array}$ & $\begin{array}{l}0.580 \\
0.001\end{array}$ & $\begin{array}{l}0.541 \\
0.001\end{array}$ & $\begin{array}{l}0.729 \\
0.001\end{array}$ & $\begin{array}{l}0.282 \\
0.064\end{array}$ & $\begin{array}{l}0.498 \\
0.001\end{array}$ & $\begin{array}{l}0.849 \\
0.001\end{array}$ & $\begin{array}{l}0.407 \\
0.006\end{array}$ & $\begin{array}{l}0.776 \\
0.001\end{array}$ & $\begin{array}{l}0.615 \\
0.001\end{array}$ & $\begin{array}{l}0.727 \\
0.001\end{array}$ & $\begin{array}{l}0.699 \\
0.001\end{array}$ & $\begin{array}{l}0.705 \\
0.001\end{array}$ \\
\hline
\end{tabular}

Bold indicates $(P<0.05)$.

pyruvate levels were comparable with published physiological values (http://dairynet.traill.uiuc.edu). FF lactate profiles were lower overall than those of Leroy et al. (2004), possibly as a result of post mortem glycolysisinduced changes in composition in the latter study. The present carbohydrate profiles compared favourably with the values reported in the human by Leese \& Lenton (1990) $(0.45,3.29$ and $6.12 \mathrm{mmol} / \mathrm{l}$ pyruvate, glucose and lactate respectively), perhaps due to the similar follicle size in both species. Antral follicles with different granulosa cell area:antral volume ratios, such as those of the mouse, have a different carbohydrate profile (Harris et al. 2003), likely due to granulosa/theca cell glycolytic metabolism (Boland et al. 1993, 1994).

Whereas pyruvate and lactate were correlated with FF volume positively and negatively respectively, glucose was not. These findings differed from those reported for human FF, possibly due to differences in theca/granulosa cell metabolism or oestrous stage at the time of sampling (Leese \& Lenton 1990). Nonetheless, glucose and lactate concentrations were significantly negatively correlated, as reported by Leese \& Lenton (1990), implying the participation of follicular glycolysis superimposition upon the flux of these carbohydrates from plasma.

\section{Amino acid profiles}

To the best of our knowledge, there is only one earlier study on the amino acid composition of bovine FF, which found qualitatively similar profiles to those given here (Guérin et al. 1995). Quantitative discrepancies may be accounted for by differences in analytical technique, smaller sample size and the absence of follicular segregation according to oestrous stage in the work of Guérin et al. (1995). Amino acid profiles were very homogeneous, independent of oestrous stage or follicle status, except for taurine, arginine and leucine, suggesting that follicle size has minor effects on amino acid levels. Although certain amino acids (glycine, alanine, proline, serine, tyrosine, glutamate and lysine) are taken up by 


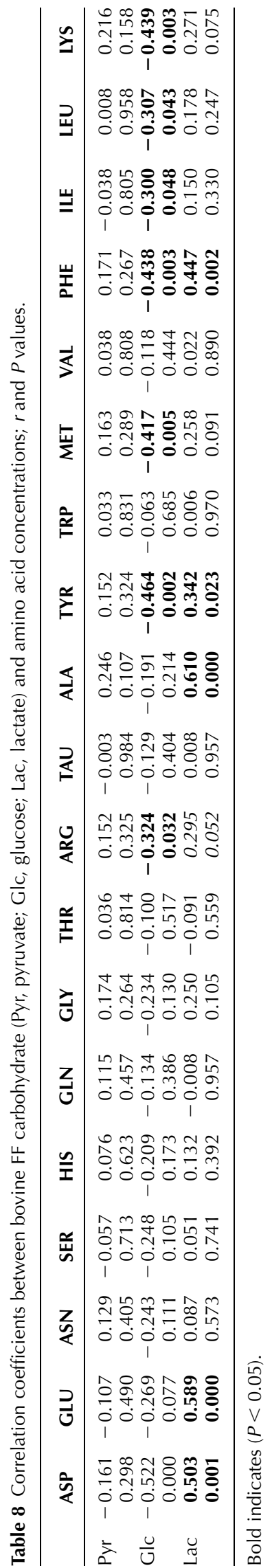

Table 9 Pyruvate, glucose and lactate concentrations in Stage IV dominant bovine ovarian follicles and maturation medium.

\begin{tabular}{lccc}
\hline & Pyruvate $(\mathrm{mmol} / \mathrm{l})$ & Glucose $(\mathrm{mmol} / \mathrm{l})$ & Lactate $(\mathrm{mmol} / \mathrm{l})$ \\
\hline Stage IV & $0.03 \pm 0.01$ & $4.84 \pm 0.14$ & $5.09 \pm 0.37$ \\
Maturation & $1.01 \pm 0.10^{* * *}$ & $5.41 \pm 0.76$ & $2.23 \pm 0.45^{* * *}$ \\
\hline
\end{tabular}

*** $P<0.001$.

granulosa cells and transferred to the oocyte (Colonna \& Mangia 1983), only serine and glutamate followed such a pattern consistently throughout the oestrous cycle. This suggests that passage of amino acids into the antrum may satisfy the requirements of developing granulosa cells. Interestingly, FF amino acid concentrations were lower than those found in the oviduct, despite having qualitatively similar profiles. Furthermore, they were comparable with plasma amino acid levels (Guérin et al. 1995, present authors, unpublished observations).

Interestingly, amino acid concentration was negatively correlated with follicular volume, significantly so in the case of aspartate, glutamate, alanine, tyrosine, methionine, phenylalanine and leucine (slightly so for arginine). This may have reflected selective mural granulosa utilisation/production, although the profile of these amino acids followed no clear pattern according to whether they are essential or non-essential. With a few very marginal exceptions, all amino acid concentrations were very significantly positively correlated with each other, suggesting that the regulatory influences that affect their profile influence them in a similar manner overall (i.e. arguing against differential metabolism).

Pyruvate and amino acid concentrations were not correlated. Although this suggests that amino acid metabolism (and, speculatively, protein synthesis) is not correlated with pyruvate production by cumulus cells, it is likely that the impact of cumulus cells to FF composition is minimal compared with mural granulosa cells, due to the large size of bovine follicles. By contrast, glucose concentration was negatively correlated with arginine, tyrosine, methionine, phenylalanine, isoleucine, leucine and lysine concentrations. Except arginine (which is nevertheless readily consumed by proliferating cells (Sakagami et al. 1998)) and tyrosine, all of these are essential amino acids and thus substrates for follicular development, like glucose. Lactate profile, on the other hand, was positively correlated with aspartate, glutamate, alanine, tyrosine and phenylalanine. Except phenylalanine, these are non-essential amino acids and can be readily produced by cells, like lactate (Salway 1996).

\section{Comparison of preovulatory FF composition with IVM medium}

Glucose concentrations were similar in FF and IVM medium, since both reflect plasma concentrations, whereas pyruvate and lactate levels differed markedly. 


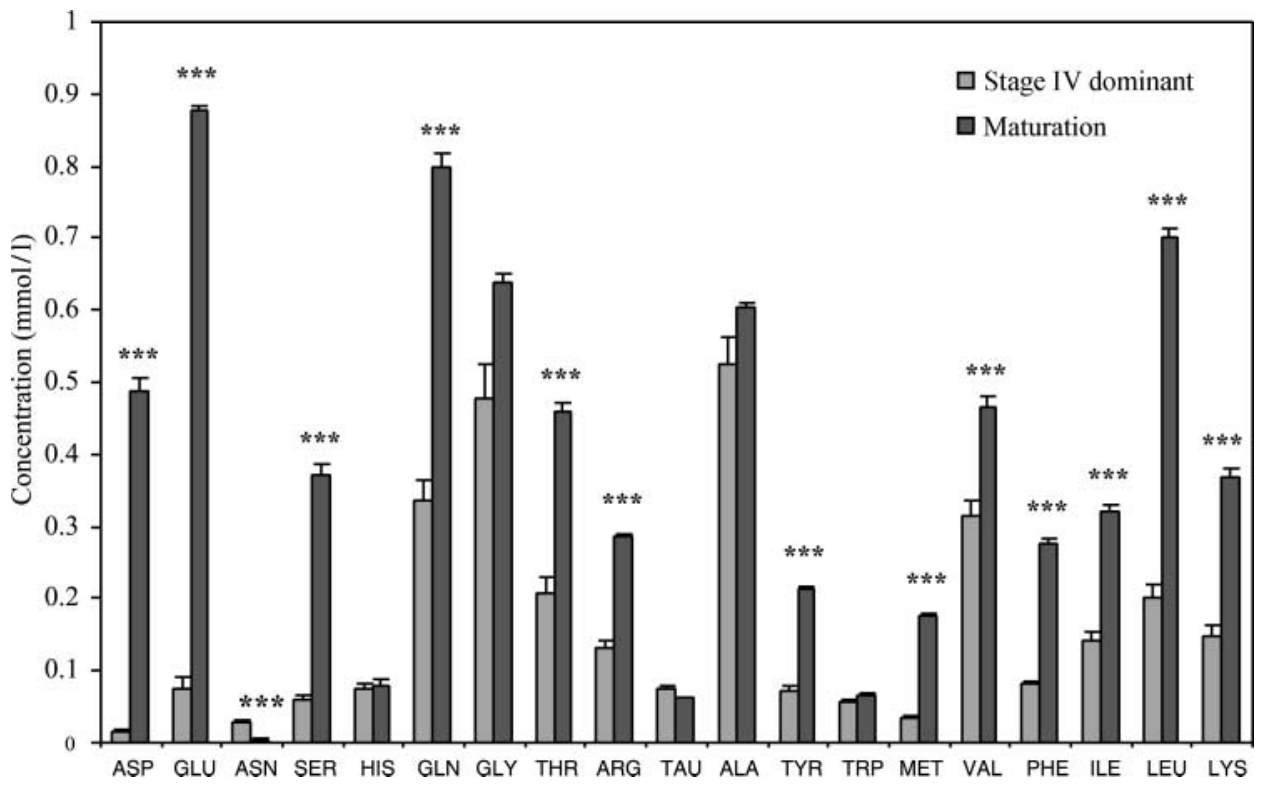

Figure 1 Amino acid profile of Stage IV dominant bovine ovarian follicles and maturation medium. $* * * P<0.001$.
The difference in pyruvate concentration is accounted for by base medium supplementation with $1 \mathrm{mmol} / \mathrm{l}$ pyruvate. Although pyruvate confers protection against oxidative stress (Salahudeen et al. 1991, O'Fallon \& Wright 1995, Morales et al. 1999, Orsi \& Leese 2001), these levels are excessive to support the nutritional requirements of the oocyte. The differences in FF and maturation medium lactate levels are accounted for by the absence of this compound in maturation medium, except for that derived from foetal calf serum. FF lactate, by contrast, is likely derived from granulosa/theca cell glycolytic metabolism (Hillier et al. 1985, Boland et al. 1993). The large disparities in pyruvate:lactate ratio between FF and IVM medium will almost certainly have a biological impact by influencing oocyte metabolism, REDOX potential (Harvey et al. 2002) and meiotic regulation through alterations in $\mathrm{pH}$ (Downs \& Mastropolo 1997).

The higher amino acid levels in maturation medium are derived from three sources: TCM-199, additional glutamine and serum. These may be beneficial by regulating metabolism (as shown for early mouse embryos (Lane \& Gardner 1998)) and protein synthesis (Kuran et al. 2002), and by conferring protection against osmotic shock (Lane 2001) and oxidative stress (Lindenbaum 1973). However, they also result in ammonium production as a result of spontaneous degradation and catabolism (Lane \& Gardner 1994, 1995, Orsi \& Leese 2004a), although this is unlikely to accumulate to toxic levels over the 24-h maturation period, since bovine OCCs are relatively tolerant to ammonium (Hammon et al. 2000a,b). The ratio of different amino acids may also be important for the oocyte. By analogy, in mammalian embryos, the free amino acid ratio - whether consumed as free molecules (Jung et al. 1998) or as proteins to be hydrolysed (Orsi \& Leese 2004b) - impacts on metabolism, gene expression, developmental potential and quality (Jung et al. 1998, Liu et al. 2002). A similar rationale may also apply to cattle oocytes during maturation. In particular, as the protein profiles of FF at different stages of oestrus and plasma differ (Spitzer et al. 1996), so will the resultant amino acid mixture obtained by hydrolysis.

The comparable osmolalities of IVM medium and FF suggest that OCCs are unlikely to be subjected to osmotic shock in the present IVM system. However, the individual concentration of different electrolytes may nonetheless play a significant role in supporting oocyte viability.

In conclusion, cycle stage and follicle status markedly impact on follicular carbohydrate and - to a lesser extent - amino acid concentrations, supporting the proposition that FF composition varies throughout the oestrous cycle (Wise 1987, Gosden et al. 1988, Spitzer et al. 1996). Preovulatory FF nutrient profile differs markedly from that of maturation medium, which may account for the poor developmental competence of IVM oocytes induced by a suboptimal IVM medium. We hope that our data will lead to the formulation of a more physiological medium for the in vitro maturation of bovine oocytes.

\section{Acknowledgements}

This work was supported by the European Commission and University of York, Burgess and White Rose studentships. The authors wish to thank Mr Peter Humpherson for advice with the amino acid analyses and the staff at Anglo Beef Processors, York for providing on-site tissue processing facilities. The authors declare that there is no conflict of interest that would prejudice the impartiality of this work.

\section{References}

Aguilar JJ, Woods GL, Miragaya MH, Olsen LM \& Vanderwall DK 2001 Effect of homologous preovulatory follicular fluid on in vitro 
maturation of equine cumulus-oocyte complexes. Theriogenology $56745-758$

Avery B, Strøbech L, Jacobsen T, Bøgh IB \& Greve T 2003 In vitro maturation of bovine cumulus-oocyte complexes in undiluted follicular fluid: effect on nuclear maturation, pronucleus formation and embryo development. Theriogenology 59 987-999.

Ayoub MA \& Hunter AG 1993 Inhibitory effect of bovine follicular fluid on in vitro maturation of bovine oocytes. Journal of Dairy Science 76 95-100.

Bøgh IB, Bézard J, Duchamp G, Baltsen M, Gérard N, Daels P \& Greve T 2002 Pure preovulatory follicular fluid promotes in vitro maturation of in vivo aspirated equine oocytes. Theriogenology $\mathbf{5 7}$ 1765-1779.

Boland NI, Humpherson PG, Leese HJ \& Gosden RG 1993 Pattern of lactate production and steroidogenesis during growth and maturation of mouse ovarian follicles in vitro. Biology of Reproduction 48 798-806.

Boland NI, Humpherson PG, Leese HJ \& Gosden RG 1994 Characterization of follicular energy metabolism. Human Reproduction $\mathbf{9}$ 604-609.

Carolan C, Lonergan P, Khatir H \& Mermillod P 1996 In vitro production of bovine embryos using individual oocytes. Molecular Reproduction and Development 45 145-150.

Chen SH, Vaught TD, Monahan JA, Boone J, Emslie E, Jobst PM, Lamborn $A E$, Schnieke A, Robertson L, Colman A, Dai $Y$, Polejaeva IA \& Ayares DL 2002 Efficient production of transgenic cloned calves using preimplantation screening. Biology of Reproduction 67 1488-1492.

Choi YH, Takagi M, Kamishita H, Wijayagunawardane MPB, Acosta TJ, Miyazawa K \& Sato K 1998 Developmental capacity of bovine oocytes matured in two kinds of follicular fluid and fertilized in vitro. Animal Reproduction Science 50 27-33.

Choudary JB, Gier HT \& Marion GB 1968 Cyclic changes in bovine vesicular follicles. Journal of Animal Science 27 468-471.

Colonna R \& Mangia F 1983 Mechanisms of amino acid uptake in cumulus-enclosed mouse oocytes. Biology of Reproduction $\mathbf{2 8}$ 797-803.

Coy P, Ruiz S, Romar R, Campos I \& Gadea J 1999 Maturation, fertilization and complete development of porcine oocytes matured under different systems. Theriogenology 51 799-812.

Dell'Aquila ME, Cho YS, Minoia P, Traina V \& Lacalandra GM 1997 Effects of follicular fluid supplementation of in vitro maturation medium on the fertilization and development of equine oocytes after in-vitro fertilization or intracytoplasmic sperm injection. Human Reproduction 12 2766-2772.

Donahue RP \& Stern S 1968 Follicular cell support of oocyte maturation: production of pyruvate in vitro. Journal of Reproduction and Fertility 17 395-398.

Downs SM \& Mastropolo AM 1997 Culture conditions affect meiotic regulation in cumulus cell-enclosed mouse oocytes. Molecular Reproduction and Development 46 551-566.

Eppig JJ \& O'Brien MJ 1998 Comparison and preimplantation developmental competence after mouse oocyte growth and development in vitro and in vivo. Theriogenology 49 415-422.

Eppig JJ, O'Brien MJ \& Wigglesworth K 1996 Mammalian oocyte growth and development in vitro. Molecular Reproduction and Development 44 260-273.

Erickson BH 1966 Development and senescence of the bovine ovary. Journal of Animal Science 25 800-805.

Espey LL \& Lipner H 1994 Ovulation. In The Physiology of Reproduction, pp 725-780. Eds E Knobil \& JD Neill. New York: Raven Press.

Galli C, Duchi R, Crotti G, Turini P, Ponderato N, Colleoni S, Lagutina I \& Lazzari G 2003 Bovine embryo technologies. Theriogenology 59 599-616.

Gérard N, Loiseau S, Duchamp G \& Seguin F 2002 Analysis of the variations of follicular fluid composition during follicular growth and maturation in the mare using proton nuclear magnetic resonance (1H NMR). Reproduction 124 241-248.
Gerritse R, Kruip TA, van Beneden TH \& Velde ER 1988 Maturation of bovine oocytes transplanted into bovine follicles of follicular and luteal phase. International Journal of Fertility 33 134-138.

Gopichandran N \& Leese HJ 2003 Metabolic characterization of the bovine blastocyst, inner cell mass, trophectoderm and blastocoel fluid. Reproduction 126 299-308.

Gosden RG, Hunter RHF, Telfer E, Torrance C \& Brown N 1988 Physiological factors underlying the formation of ovarian follicular fluid. Journal of Reproduction and Fertility 82 813-825.

Guérin P, Gallois E, Croteau S, Revol N, Maurin F, Guillaud J \& Ménézo Y 1995 Techniques de récolte et aminogrammes des liquides tubaire et folliculaire chez les femelles domestiques. Revue de Médecine Vétérinaire 146 805-814.

Gull I, Geva E, Lerner-Geva L, Lessing J, Wolman I \& Amit A 1999 Anaerobic glycolysis. The metabolism of the preovulatory human oocyte. European Journal of Obstetrics, Gynecology and Reproductive Biology 85 225-228.

Hammon DS, Wang S \& Holyoak GR 2000a Ammonia concentration in bovine follicular fluid and its effect during in vitro maturation on subsequent embryo development. Animal Reproduction Science 58 1-8.

Hammon DS, Wang S \& Holyoak GR 2000b Effects of ammonia during different stages of culture on development of in vitro produced bovine embryos. Animal Reproduction Science 59 23-30.

Harris SE, Leese HJ, Picton HM \& Orsi NM 2003 Nutrient concentrations in oestrous mouse ovarian follicles, oviduct and uterus. Reproduction 3092 (Abstract).

Harvey AJ, Kind KL \& Thompson JG 2002 REDOX regulation of early embryo development. Reproduction 123 479-486.

Hillier SG, Purohit A \& Reichert LE Jr 1985 Control of granulosa cell lactate production by follicle-stimulating hormone and androgen. Endocrinology 116 1163-1167.

Ireland JJ, Murphee RL \& Coulson PB 1980 Accuracy of predicting stages of bovine estrous cycle by gross appearance of the corpus luteum. Journal of Dairy Science 63 155-160.

Jimena P, Castilla JA, Peran F, Ramirez JP, Gil T, Mozas J, Martinez L \& Herruzo A 1993 Distribution of free amino acids in human preovulatory follicles. Hormone and Metabolism Research 25 228-230.

Jung YG, Sakata T, Lee ES \& Fukui Y 1998 Amino acid metabolism of bovine blastocysts derived from parthenogenetically activated or in vitro fertilized oocytes. Reproduction, Fertility and Development 10 279-287.

Krisher RL \& Bavister BD 1998 Responses of oocytes and embryos to the culture environment. Theriogenology 49 103-114.

Kruip AM \& Dieleman SJ 1982 Macroscopic classification of bovine follicles and its validation by micromorphological and steroid biochemical procedures. Reproduction, Nutrition and Development 22 465-473.

Kubota C, Yang X, Dinnyes A, Todoroki J, Yamakuchi H, Mizoshita K, Inohae S \& Tabara N 1998 In vitro and in vivo survival of frozen-thawed bovine oocytes after IVF, nuclear transfer, and parthenogenetic activation. Molecular Reproduction and Development $51281-286$.

Kuran M, Robinson JJ, Brown DS \& McEvoy TG 2002 Development, amino acid utilization and cell allocation in bovine embryos after in vitro production in contrasting culture systems. Reproduction 124 155-165.

Lane M 2001 Mechanisms for managing cellular and homeostatic stress in vitro. Theriogenology 55 225-236.

Lane M \& Gardner DK 1994 Increase in postimplantation development of cultured mouse embryos by amino acids and induction of fetal retardation and exencephaly by ammonium ions. Journal of Reproduction and Fertility 102 305-312.

Lane M \& Gardner DK 1995 Removal of embryo-toxic ammonium from the culture medium by in situ enzymatic conversion to glutamate. Journal of Experimental Zoology 271 356-363.

Lane M \& Gardner DK 1998 Amino acids and vitamins prevent culture-induced metabolic perturbations and associated loss of 
viability of mouse blastocysts. Human Reproduction 13 991-997.

Lebedeva IY, Denisenko VY, Lebedev VA \& Kuzmina TI 1998 Prolactin in follicular fluid and intracellular store calcium in follicular cells are related to morphological signs of ovarian follicle atresia in cows: work in progress. Theriogenology 49 509-519.

Leese HJ \& Barton AM 1985 Production of pyruvate by isolated mouse cumulus cells. Journal of Experimental Zoology 234 231-236.

Leese HJ \& Lenton EA 1990 Glucose and lactate in human follicular fluid: concentrations and interrelationships. Human Reproduction 5 915-919.

Leroy JLMR, Vanholder T, Delanghe JR, Opsomer G, Van Soom A, Bols PEJ \& de Kruif A 2004 Metabolite and ionic composition of follicular fluid from different-sized follicles and their relationship to serum concentrations in dairy cows. Animal Reproduction Science 80 201-211.

Lim JM, Lee BC, Chung HM, Ko JJ, Park SE, Cha KY \& Hwang WS 1999 In vitro maturation and in vitro fertilization of bovine oocytes cultured in a chemically defined, protein-free medium: effects of carbohydrates and amino acids. Reproduction, Fertility and Development 11 127-132.

Lindenbaum A 1973 survey of naturally occurring chelating ligands. Advances in Medical Biology 40 67-77.

Liu H-C, He Z \& Rosenwaks Z 2002 Global gene expression profiles of individual blastocysts cultured in medium with various compositions of amino acids. Fertility and Sterility 78 S225-S226 (Abstract).

Lonergan P, Rizos D, Ward F \& Boland MP 2001 Factors influencing oocyte and embryo quality in cattle. Reproduction, Nutrition and Development 41 427-437.

McNatty KP 1978 Follicular fluid. In The Vertebrate Ovary, pp 215-259. Ed. RE Jones. New York: Plenum Press.

Morales H, Tilquin P, Rees JF, Massip A, Dessy F \& Van Langedonckt A 1999 Pyruvate prevents peroxide-induced injury of in vitro preimplantation bovine embryos. Molecular Reproduction and Development 52 149-157.

Nagai T 2001 The improvement of in vitro maturation systems for bovine and porcine oocytes. Theriogenology 55 1291-1301.

Nakazawa T, Ohashi K, Yamada M, Shinoda S, Saji F, Murata Y \& Araki H 1997 Effect of different concentrations of amino acids in human serum and follicular fluid on the development of one-cell mouse embryos in vitro. Journal of Reproduction and Fertility $\mathbf{1 1 1}$ 327-332.

O'Fallon JV \& Wright RW Jr 1995 Pyruvate revisited: a non-metabolic role for pyruvate in preimplantation embryo development. Theriogenology 43288.

Orsi NM \& Leese HJ 2001 Protection against reactive oxygen species during mouse preimplantation development: role of EDTA, oxygen tension, catalase, superoxide dismutase and pyruvate. Molecular Reproduction and Development 59 44-53.

Orsi NM \& Leese HJ 2004a Ammonium exposure and pyruvate affect the amino acid metabolism of bovine blastocysts. Reproduction $127131-140$.
Orsi NM \& Leese HJ 2004b Amino acid metabolism of preimplantation bovine embryos cultured with bovine serum albumin or polyvinyl alcohol. Theriogenology 61 561-572.

Partridge RJ \& Leese HJ 1996 Consumption of amino acids by bovine preimplantation embryos. Reproduction, Fertility and Development 8 945-950.

Rieger D \& Loskutoff NM 1994 Changes in the metabolism of glucose, pyruvate, glutamine and glycine during maturation of cattle oocytes in vitro. Journal of Reproduction and Fertility 100 257-262.

Rodriguez H, Torres C, Valdes X, Guerra H, Pastor LM, Maccallini G \& Bustos-Obregon E 2001 The acrosomic reaction in stallion spermatozoa: inductive effect of the mare preovulatory follicular fluid. Biocell 25 115-120.

Sakagami H, Satoh M, Yokote Y, Takano H, Takahama M, Kochi M \& Akahane K 1998 Amino acid utilization during cell growth and apoptosis induction. Anticancer Research 18 4303-4306.

Salahudeen AK, Clark EC \& Nath KA 1991 Hydrogen peroxideinduced renal injury - a protective role for pyruvate in vitro and in vivo. Journal of Clinical Investigation 88 1886-1893.

Salway JG 1996 In Metabolism at a Glance, edn 2. Oxford: Blackwell Science.

Sirard MA \& First NL 1988 In vitro inhibition of oocyte nuclear maturation in the bovine. Biology of Reproduction 39 229-234.

Smitz J \& Cortvrindt R 1999 Oocyte in-vitro maturation and follicle culture: current clinical achievements and future directions. Human Reproduction 14 145-161.

Spitzer D, Murach KF, Lottspeich F, Staudach A \& Illmensee K 1996 Different protein patterns derived from follicular fluid of mature and immature human follicles. Human Reproduction 11 798-807.

Takagi M, Choi YH, Kamishita H, Ohtani M, Acosta TJ, Wijayagunawardane MP, Miyamoto A, Miyazawa K, Sato K \& Sato E 1998 Evaluation of fluids from cystic follicles for in vitro maturation and fertilization of bovine oocytes. Theriogenology 50 307-320.

Wang Y, Storeng R, Dale PO, Abyholm T \& Tanbo T 2001 Effects of follicular fluid and steroid hormones on chemotaxis and motility of human spermatozoa in vitro. Gynaecology and Endocrinology 15 286-292.

Watson AJ, De Sousa P, Caveney A, Barcroft LC, Natale D, Urquhart J \& Westhusin ME 2000 Impact of bovine oocyte maturation media on oocyte transcript levels, blastocyst development, cell number, and apoptosis. Biology of Reproduction 62 355-364.

Wise T 1987 Biochemical analysis of bovine follicular fluid: albumin, total protein, lysosomal enzymes, ions, steroids and ascorbic acid content in relation to follicular size, rank, atresia classification and day of oestrous cycle. Journal of Animal Science 64 1153-1169.

Zuelke KA \& Brackett BG 1993 Increased glutamine metabolism in bovine cumulus cell-enclosed oocytes after in vitro maturation with luteinizing hormone. Biology of Reproduction 48 815-820.

Received 12 August 2004

First decision 4 October 2004

Revised manuscript received 12 October 2004

Accepted 1 November 2004 